\title{
Age Differences in Strategy Shift: Retrieval Avoidance or General Shift Reluctance?
}

\author{
David J. Frank, \\ Department of Psychology, The University of North Carolina at Greensboro \\ Dayna R. Touron, and \\ Department of Psychology, The University of North Carolina at Greensboro \\ Christopher Hertzog \\ School of Psychology, Georgia Institute of Technology
}

\begin{abstract}
Previous studies of metacognitive age differences in skill acquisition strategies have relied exclusively on tasks with a processing shift from an algorithm to retrieval strategy. Older adults' demonstrated reluctance to shift strategies in such tasks could reflect either a specific aversion to a memory retrieval strategy or a general, inertial resistance to strategy change. Haider and Frensch's (1999) alphabet verification task (AVT) affords a non-retrieval-based strategy shift. Participants verify the continuation of alphabet strings such as D E F G [4] L, with the bracketed digit indicating a number of letters to be skipped. When all deviations are restricted to the letter-digitletter portion, participants can speed their responses by selectively attend only to that part of the stimulus. We adapted the AVT to include conditions which promoted shift to a retrieval strategy, a selective attention strategy, or both strategies. Item-level strategy reports were validated by eye movement data. Older adults shifted more slowly to the retrieval strategy but more quickly to the selective attention strategy than young adults, indicating a retrieval-strategy avoidance. Strategy confidence and perceived strategy difficulty correlated with shift to the two strategies in both age groups. Perceived speed of responses with each strategy specifically correlated with older adults' strategy choices, suggesting that some older adults avoid retrieval because they do not appreciate its efficiency benefits.
\end{abstract}

\section{Keywords}

Strategies; Skill Acquisition; Metacognition

\begin{abstract}
Many processes in everyday life become more efficient with practice. Improvements often involve a shift from a slow and effortful process, such as taking out your social security card to find the number, to a more efficient and often automatic process, like retrieving that number from memory.

As people age their rate of acquiring new information slows, as does their rate of retrieving newly learned associations (Kausler, 1994; Ratcliff, Thapar, \& McKoon, 2011; Touron, Hoyer, \& Cerella, 2001). The use of newly learned information also changes with age. Older adults switch from algorithm to retrieval strategies more slowly than do younger adults, a finding observed on a number of tasks including two-digit multiplication (Lamson \&
\end{abstract}

\footnotetext{
Correspondence concerning this article should be addressed to Dayna R. Touron, Associate Professor of Psychology, Department of Psychology, 296 Eberhart Bldg. PO Box 26170, The University of North Carolina at Greensboro, Greensboro, NC 27402-6170, d_touron@uncg.edu.
} 
Rogers, 2008), alphabet arithmetic (Hoyer, Cerella, \& Onyper, 2003), novel symbolic arithmetic (Touron et al., 2001), reading comprehension (Rawson \& Touron, 2009), and noun-pair associate learning (Rogers \& Gilbert, 1997; Rogers, Hertzog \& Fisk, 2000; Touron, 2006; Touron \& Hertzog, 2004a; 2004b; Touron, Hertzog, \& Frank, 2010; Touron, Swaim, \& Hertzog, 2007).

Both learning ability and metacognition play a role in older adults' delayed strategy shift (Rogers et al., 2000; Strayer \& Kramer, 1994; Touron \& Hertzog, 2004a; 2004b). Older adults' use of retrieval strategies is substantially delayed even after they have learned the requisite information (Hines et al., 2012; Touron \& Hertzog, 2004a). Retrieval use is also influenced by individuals' response criteria and memory confidence (Touron \& Hertzog, $2004 b$ ), and older adults' retrieval shift is hastened by performance incentives (Touron \& Hertzog, 2009; Touron et al., 2007). Extensive pre-learning of the paired-associate items eliminates age differences in retrieval shift in the noun-pair learning task, but mixing prelearned and new items in the same stimulus set causes older adults to avoid retrieval use on pre-learned items (Hines et al., 2012).

These outcomes align with other evidence that strategy choice is influenced by top-down mechanisms (e.g., Bourne, Raymond, \& Healy, 2012; Haider, Frensch, \& Joram, 2005). However, it is unknown whether old adults' delayed strategy shift would be generically observed in skill acquisition tasks or instead would be seen primarily when the task requires shifting to a memory retrieval strategy. The purpose of this study was to shed light on this issue of general versus specific strategy shift avoidance among older adults.

\section{Retrieval Avoidance vs. General Shift Reluctance}

Several metacognitive mechanisms may help to explain older adults' delayed retrieval shift. Older adults who are the most retrieval avoidant report less confidence in their ability to use retrieval-based strategies (e.g., Touron \& Hertzog, 2004a, 2004b). Confidence in the accuracy of associative recognition memory responses is lower in older adults and has a weaker association with recognition memory accuracy; in turn, recognition memory confidence judgments predict slower shifts to retrieval by older adults (Hertzog \& Touron, 2011). Hertzog, Touron, and Hines (2007) showed that older adults underestimate the amount of time required to perform an algorithm and that the degree of this underestimation predicted greater retrieval reluctance. Thus, older adults may persevere in using an algorithmic strategy in part because they do not accurately monitor the relative costs and benefits of the different strategies they attempt.

It is also possible that older adults are generically less likely to shift strategies even when they do comprehend the potential benefits of using a more effective or more efficient strategy (Lemaire, 2010). According to a general shift reluctance hypothesis, older adults are more ingrained in a habitual processing approach, and are generically reluctant to change that approach (Hertzog, Price, \& Dunlosky, 2008; Spieler, Mayr, \& Lagrone, 2006). For example, older adults are more likely to maintain use of a relatively ineffective associative learning strategy, rote repetition, after instructed task experience contrasting repetition with the more effective interactive imagery strategy (Hertzog, Price, \& Dunlosky, 2012). Changing strategies may also invoke switch costs (increased RTs for adjacent trials involving different tasks compared those involving the same task) akin to those seen in task switching studies (Lemaire \& Lecacheur, 2010). Older adults sometimes show larger switch costs relative to young adults (Kray \& Lindenberger, 2000; Mayr, 2001; but see Wasylyshyn, Verhaeghen, \& Sliwinski, 2011; Whitson, Karayanidis, \& Michie, 2012), and thus may be less likely to switch tasks voluntarily (Arrington \& Logan, 2004; 2005). Spieler and colleagues (2006) demonstrated that older adults continued to fixate previously 
necessary but now irrelevant task cues, and that this continued processing of newly irrelevant task cues increased their response latencies. Although multiple interpretations of this effect are possible, it could indicate a kind of global strategic inertia-sticking with what seems to work, even when it is no longer necessary. This behavior depended upon the physical presence of the cue (see also Touron et al., 2010); when the cue was removed older adults ceased to fixate the cue region and decreased their RTs.

Because all available research to date has examined shift from algorithm to retrieval it is not currently possible to discriminate retrieval shift avoidance from general behavioral inertia. This study explores the specific versus general nature of older adults' shift reluctance using a single task that allows for a shift to either retrieval-based or non-retrieval-based strategies.

\section{Alphabet Verification Task}

The alphabet verification task (AVT) developed by Haider and Frensch (1996) has been used to study skill acquisition in young adults. It involves a shift to a more efficient strategy that is not based on memory retrieval. In the AVT, participants verify the consistency of stimuli with the standard rule of alphabetic order. The AVT presents a series of letters interrupted by a bracketed number representing a number of letters to be skipped before continuing the string (e.g., B C D [4] I). We refer to the critical letter-digit-letter sequence (D [4] I in the prior example) as the triplet. Haider and Frensch (1996) instructed participants that deviations from alphabetical correctness could occur at any point in the string inside the triplet (e.g., B C D [4] J, where J is alphabetically deviant because I should follow D [4]) or outside the triplet (e.g., B D E [4] J, where D is deviant because C should follow B).

In the actual experiment deviations only occurred inside the triplet, so participants could respond more quickly if they only attended to and expanded the triplet portion of the string. Haider and Frensch (1996) refer to this as a selective attention strategy, because one attends only to the relevant portion of the stimulus. Some participants spontaneously discovered and employed the selective attention strategy with resulting benefits in AVT RTs.

Selective attention strategies can be observed in expert behavior outside the laboratory (Haider \& Frensch, 1996). For example, expert chess players selectively attend only to relevant chess pieces when considering a move, whereas novice chess players tend to scan the entire board before considering a move (Frensch \& Sternberg, 1991). Thus, selective attention is comparable to retrieval in that both strategies reduce RTs, reduce effort, and are used in everyday life.

\section{Goals and Hypothesis}

The present study adapted the AVT task by creating between-subjects conditions which vary strategy shift possibilities. In a selective attention condition, Haider and Frensch's (1996) selective attention strategy and full computation were possible. In a retrieval condition, retrieval and full computation were possible. In a choice condition, retrieval, selective attention, and full computation strategies were possible. We expected young adults to shift to the more efficient strategy whenever possible. Consistent with past research, we expected older adults to shift to a retrieval strategy more slowly compared to young adults. Slower shift to both retrieval and selective attention by older adults (more computation use) would indicate general shift reluctance. Slower shift by older adults to the retrieval strategy alone would indicate specific retrieval reluctance. However, whether older adults in the choice condition will choose selective attention or retrieval may depend on the relative efficiency of the two strategies for them. 
We measured strategies in the modified AVT task by requiring strategy reports after each trial response. Performance and eye-tracking data validated retrieval reports in the noun-pair lookup task (Touron et al., 2010), but self-reports have not previously been used to measure strategies in the AVT task. To validate strategy reports in the current experiment we used a combination of RT and eye-tracking data. Regarding the latter, more gazes made to longer strings (such regular increases in processing are referred to as addend effects), should be present when participants compute, but not when they use selective attention (Haider \& Frensch, 1996; 1999) or retrieval. To foreshadow, eye-tracking and RT data supported the validity of the strategy reports.

\section{Methods}

\section{Design and Participants}

The primary between-subject independent variables were age (old, young) and condition (retrieval, selective attention, or choice). Eighty-five young adults (ages 18-21) and 67 older adults (ages 60-75) were randomly assigned to strategy conditions. ${ }^{1}$ Young adults were psychology students and received course credit for participation. Older adults were recruited from the community and received a modest honorarium of $\$ 30$ for participation. All participants were screened for near visual acuity of at least 20/50 and older adults were prescreened for health issues. Participants diagnosed with dementia or memory loss or taking medications that affect memory or alertness were excluded from the study. Participants reporting factors which complicate eye tracking, including glaucoma, cataracts, colored contacts, or transitional lenses were also excluded from the study.

Twenty-three young and seven older adults were excluded from analysis due to poor performance (less than $70 \%$ accuracy), computer errors, indications of disbelief, or misunderstanding of instructions. ${ }^{2}$ Participants removed from analysis were replaced, resulting in roughly twenty participants per age $\mathrm{X}$ condition cell.

Participant characteristics can be found in Table 1. The mean age for older adults was greater in the choice condition compared to the other two conditions. Compared to young, older adults were more educated, rated themselves as healthier, took more medications, had higher vocabulary scores, completed fewer items on the digit symbol test (a test of processing speed), and performed worse on the digit symbol recall test (a test of implicit associative memory). This pattern is similar to that found in other age-related skill acquisition studies (e.g., Touron \& Hertzog, 2004a; 2004b) and in cognitive aging research in general.

\section{Stimuli and Apparatus}

Participants were seated in front of a Dell computer with LCD monitor at a distance of roughly $61 \mathrm{~cm}$. Screen resolution was set to $1024 \times 768$. Participants responded via key presses to stimuli presented via a Visual Basic 6.0 program. Eye movements were recorded via an Applied Sciences Laboratories head-mounted eye-tracker (model H6HS with eyehead integration) recorded at a sampling rate of $120 \mathrm{~Hz}$. Letter strings appeared in 22 point

\footnotetext{
${ }^{1}$ We also included a control condition in which no strategy shift was possible. However this condition does not address the primary research question and is not discussed further.

${ }^{2}$ Two of the excluded young adults in the retrieval condition rarely used the retrieval strategy and endorsed beliefs about the retrieval strategy inconsistent with those of their peers. It is unknown whether these individuals are merely outliers or represent a sub-group of young adults. No similar subgroup of young adults has been seen in our previous strategy shift research, nor did they exist in the present choice condition. The disparity in young and older adult exclusion was almost entirely accounted for by greater mistrust in the instructions by younger adults.
} 
bolded courier new font (see Figure 1). The individual letters in the stimuli were $3.8 \mathrm{~cm}$ apart, producing a visual angle of roughly $0.56^{\circ}$ per letter and $3.6^{\circ}$ between letters.

The stimulus set consisted of 333 AVT strings of varied length. True strings contained an alphabetically correct triplet preceded by $0-4$ letters in correct alphabetical order (all five possible lengths for Phase 1; Phase 2 and 3 included only lengths $0,+2$, and +4 ). The bracketed number in each string was either a 4 or 5 ; which was balanced across string lengths and blocks. False strings varied by condition, containing one point of deviation from alphabetical order either in the triplet and/or in the preceding 1-4 letters.

In all phases error feedback was provided after every trial via a 1,000ms "ERROR" message and percent correct and mean RT were provided after each block. Each trial was preceded by a $500 \mathrm{~ms}$ fixation cross displayed in the center of the screen; participants responded to the question "Is the following string alphabetically correct?" via key press using the '.' and '/' keys labeled ' $\mathrm{Y}$ ' and ' $\mathrm{N}$ ' respectively to indicate yes or no. Between blocks participants were offered a short rest break, and the eye-tracker was recalibrated if necessary.

\section{Procedures}

All participants first completed a consent form followed by a computerized demographics questionnaire containing health and lifestyle information, the Lighthouse Test of Near Visual Acuity (Bailey, 1978), the Shipley Vocabulary Test (Zachary, 1986), and WAIS-R Digit-Symbol subtest (Wechsler, 1981). After a short break, participants completed a 9-point calibration of the eye-tracker followed by the AVT computer task.

Phase 1-Phase 1 required all participants to develop the habit of processing the entire AVT string. Participants first received standard AVT instructions followed by an instruction quiz until the quiz was completed error-free and the participants indicated that they understood the instructions. In three blocks of 30 AVT trials, deviations occurred both inside and outside the triplet. Phase 1 strings were not repeated throughout the experiment.

Phase 2-To control for expected age differences in incidental learning of the AVT stimuli for Phase 3, all participants completed a pre-learning phase in which they memorized six strings via a study-test procedure. Training continued until participants were accurate on at least 11 of 12 trials within a test block. Each test block contained one true and one false instance of each string. Two strings of each length were used to prevent responding based on length alone. Participants memorized the strings using only the first and last letters to ease memorization and prohibit computation. Memorizing the first and last letter pairings was possible because any deviation in a string will invariably alter the last letter (e.g., when skipping a letter in the non-triplet the last letter becomes one letter further in the alphabet). Criterion testing presented only the first and last letters of the strings (with interior letters and triplets filled with placeholders; see Appendix).

Phase 3-In Phase 3, participants performed six blocks of 24 AVT trials. For all conditions, each block contained eight strings for each of the three lengths (triplet plus 0,2 , or 4 letters), half true and half false. In the condition affording only the selective attention strategy, strings were non-repeated and pseudo-randomly selected, and false strings contained deviations only in the triplet portion of the string. In the condition affording only the retrieval strategy, true strings were those pre-learned during Phase 2 (repeated twice per block) and false strings were deviant versions of those true strings with deviations either inside or outside the triplet (deviant versions of each string were also presented twice per block but were not the same deviant version each time (e.g., B C D F G [4] L and B C D E F [5] M). Critically for the eye-movement validation of strategy reports, deviant strings never 
ended with the same letter as their corresponding correct string (as described above for Phase 2), so that participants could verify the strings from memory by inspecting only the first and last letters. The choice condition afforded both retrieval and selective attention strategies by including elements relevant to retrieval (all true strings were those from Phase 2 each repeated twice per block) and selective attention (deviant versions of those prelearned strings contained errors only in the triplet) conditions such that participants could use either strategy. In each condition following every trial, participants indicated via keypress the strategy they just used, using the ' $z$,' ' $x$,' 'c,' and, for the choice condition, ' $v$ ' keys. These keys were relabeled with the first letter of each available strategy (e.g. $\mathrm{C}=$ compute, $\mathrm{T}=$ triplet computation, $\mathrm{M}=$ memory, and $\mathrm{O}=\mathrm{other}$ ), with computation always being first (mapped to the ' $z$ ' key), followed by triplet computation/memory, and other always being last. In the choice condition the triplet computation and memory strategies were mapped to the ' $x$ ' and 'c' keys with their order counterbalanced across participants.

Prior to Phase 3, each participant read and was quizzed on condition-specific instructions which presented the strategy options (computation, selective attention and/or retrieval) and how to complete the strategy probes. Because all participants began Phase 3 having memorized the repeated strings (retrieval and choice condition) and having full awareness of the available strategies, delayed shift should be due primarily to metacognitive factors or inertia rather than differences in learning or strategy discovery.

Post-task questionnaire-Following the AVT for all conditions, participants completed a post task questionnaire which asked about task and strategy performance and beliefs which varied by condition to reflect available strategy options.

\section{Results}

Dependent variables included (1) gaze count (the number of gazes to the non-triplet region), (2) number of blocks to reach Phase 2 pre-learning criteria, (3) Phase 3 strategy probe responses (\% computation, retrieval, selective attention, or other), and (4) RTs. Because the assumption of sphericity did not hold, Greenhouse-Geisser corrected $p$-values were used for within-subjects comparisons. Accuracy was generally at or near ceiling for both age groups and all strategies and will not be discussed further.

Phase 1 gaze and RT data were as expected, with longer RTs and more gazes to longer strings (Haider \& Frensch, 1999) and no age differences. Since these data do not inform the primary question of interest they are not described in detail. There were no differences between young and older adults in the number of blocks required to reach the prelearning criterion in Phase 2 (as in Hines et al., 2012, Experiment 1; but see Touron \& Hertzog, 2004, and Touron et al., 2010), $t(123)=1.04, p=.302\left(M_{\text {young }}=1.95, S E_{\text {young }}=0.19 ; M_{\text {old }}=\right.$ $\left.1.70, S E_{\text {old }}=0.14\right)$. For Phase 3 analyses, we first compare strategy shift data, then use RTs and gaze data to demonstrate strategy benefits and strategy report validity. Lastly, we examine metacognitive influences on strategy choice.

\section{Self-reported Strategy Use}

Strategy use was computed as percentage of trials for which retrieval/selective attention strategies were used (excluding "other" reports, which removed $1.46 \%$ of trials).

Comparison of retrieval and choice condition strategy use-We first examined retrieval use in the retrieval and choice conditions to replicate previous findings of older adults' retrieval reluctance. Because "other" responses were removed prior to calculating percent retrieval use, retrieval use is thus the percent retrieval use out of all "non-other" responses, with the residual strategy use being a combination of computation and selective 
attention use. Percentage retrieval use was examined via a 2 (age: young, old) X 2 (condition: retrieval, choice) GLM which allowed us to test for both age differences in retrieval use as well as to formally test whether the option of an alternative non-retrieval strategy (selective attention) decreased older adults retrieval use in the choice condition. A main effect of age $F(1,77)=4.59, M S E=3,942, p=.035, d=0.44$, resulted from old using retrieval less compared to young. The main effect of condition, $F(1,77)=1.14, M S E=981$, $p=.288$, and the Age X Condition interaction, $F<1$, were not reliable suggesting no condition differences in retrieval use (see Figure 2). Thus we found the typical older adult retrieval reluctance, which was unaffected by the presence of an alternative (selective attention) strategy. Further analyses of retrieval use in the choice condition indicate that participants were more likely to report the retrieval strategy than either full computation or selective attention, which did not differ. ${ }^{3}$

General vs. retrieval specific shift reluctance-Having established older adults' retrieval reluctance, we compared the retrieval and selective attention conditions with a 2 (age: young, old) X 2 (condition: retrieval, selective attention) GLM for retrieval/selective attention use. Because "other" responses were removed prior to calculating percent strategy use, strategy use is thus the percent retrieval/selective attention use out of all "non-other" responses with the residual strategy use being computation use. In the selective attention condition, we also removed triplet-only strings before computing percent selective attention use because for these strings the selective attention and computation strategies are the same. A retrieval-specific shift reluctance account predicts that older adults will use less retrieval compared to young adults but will not use less selective attention compared to young adults (Age x Condition interaction). A general shift reluctance account predicts that older adults will use less retrieval and less selective attention compared to young adults (main effect of age only). A combined account would predict that older adults would be reluctant to use either strategy (compared to young adults), but that this reluctance would be greater for the retrieval strategy.

The main effects of age, $F(1,76)=1.51, M S E=1,439, p=.224$, and condition, $F(1,76)=$ $3.76, M S E=3,596, p=.056$, were not reliable (see Figure 2), with the former outcome arguing against a general shift reluctance account. An Age X Condition interaction $F(1,76)$ $=11.48, M S E=10,973, p=.001$, resulted from young adults using the selective attention strategy less often compared to older adults, $t(40)=3.77, p<.001, d=0.91$, whereas the opposite trend was found for retrieval use, $t(38)=1.51, p=.135$ (Figure 2). Although older adults used selective attention more often compared to retrieval, this $10 \%$ difference was not reliable, $t(40)=1.02, p=.309, d=0.44$, but that outcome may reflect a lack of power $(1-\mathrm{b}$ $=.27$ for a population effect size of 0.44 ).

Summary-Older adults were reluctant to use retrieval but not selective attention when full computation was the only alternative strategy, arguing for a retrieval reluctance account and against a general shift reluctance account. The presence of a second non-retrieval alternative did not further reduce older adults' retrieval use, suggesting that the selective attention strategy was less appealing when contrasted with a retrieval strategy and may have

\footnotetext{
${ }^{3} \mathrm{~A} 2$ (age: young, old) X 2 (strategy: selective attention, computation) repeated measures GLMs revealed that non-retrieval use in the choice condition was split equally between computation and selective attention; the main effects of age, $F(1,40)=1.71, M S E=855, p$ $=.198$, strategy, $F<1$, and the Age X Strategy interaction, $F<1$, were not reliable. Comparable analyses for comparing selective attention/computation use with retrieval use revealed that retrieval use was higher than either selective attention (main effect of strategy, $F(1,40)=72.72, M S E=91169, p<.001, d=2.21$ ) or computation use (main effect of strategy, $F(1,40)=42.11, M S E=$ $1738, p<.001, d=2.19$ ) and did not differ by age; main effects of Age and Age X Strategy interactions were not reliable, $p s>.22$. Prior to conducting the above analyses we removed triplet only strings as the selective attention and computation strategies are the same for these strings.
} 
only been adopted in the choice condition by those individuals who were unwilling to use retrieval.

\section{Strategy Benefits and Strategy Report Validation}

Participants must process each letter in the string when computing, but must process only the triplet when using selective attention (Haider \& Frensch, 1999) and must process only the first and last letters when using retrieval. Thus, strings with a longer non-triplet region should result in more non-triplet gazes when computing but not when using selective attention or retrieval. String length effects occur only for true strings when a computation strategy is used (participants could respond to false strings immediately after detecting an error in any portion of the string; Haider \& Frensch, 1999). Response times (RTs) should also be faster for retrieval (e.g., Touron \& Hertzog, 2004a; 2004b) and selective attention (Haider \& Frensch, 1996) compared to computation. Incorrect responses include accidental key presses and faulty processes that are not of present interest. Thus, we examine gaze counts only to the non-triplet region and gaze counts and RTs only for true strings with correct responses.

Phase 3 gaze counts and RTs were examined separately for each condition. To minimize the effects of noise and missing data due to low strategy use, we compare computation gaze counts/RTs in the first three blocks to retrieval/selective attention gaze counts/RTs in the last three blocks, but restrict these analyses to participants who used each available strategy at least once. This restriction excluded six young and five older adults in the retrieval condition, and two young and six older adults in the selective attention condition. Gaze and RT data were not examined for the choice condition as too few participants used all three strategies, however the pattern of means was generally similar to the selective attention and retrieval conditions.

Gaze counts-For gaze data, areas of interest (AOIs) were defined by a $5.08 \mathrm{~cm}$ square box around each element in the AVT string, producing a $1.3^{\circ}$ margin of error around each stimulus letter/number (see Figure 1). Stable eye position within an AOI constituted a fixation. A gaze was defined as the time between the onset of the first fixation within a given AOI and the onset of the first fixation outside that AOI (be it in a different AOI or a blank region of the screen). Pupil diameters of zero lasting more than $100 \mathrm{~ms}$ were considered blinks and not analyzed. Participants were removed from gaze analyses if more than $30 \%$ of their gazes occurred outside our AOIs (25 young and 15 older adults). For each participant, individual blocks were removed if more than $30 \%$ of gazes occurred outside the AOIs or if the technician noted inadequate tracking ( $6 \%$ of all blocks).

Non-triplet gaze counts were examined using a 2 (age: young, old) X 2 (length: $+2,+4) \times 2$ (strategy: computation, retrieval/selective attention) model. SAS Proc Mixed (Littell, Milliken, Stroup, \& Wolfinger, 2000) was used to account for missing data as not every strategy was used across each string length.

Retrieval condition: Non-triplet gaze counts for the retrieval condition are shown in Figure 3 (left panel). The main effect of age was not reliable, $F(1,20)=2.83, p=.108$. As predicted, a main effect of strategy, $F(1,20)=66.69, p<.001, d=1.42$, resulted from more gazes being made with self-reported computation compared to self-reported retrieval. This finding supports the validity of the strategy reports as participants must gaze all letters in order to compute but need only gaze the first and last letters when retrieving. A main effect of length resulted from fewer gazes on shorter strings, $F(1,20)=14.55, p=.001, d=0.40$.

The main effect of strategy was qualified by a reliable Age X Strategy interaction, $F(1,20)=$ $4.67, p=.043$, as well as a marginal Length $\mathrm{X}$ Strategy effect, $F(1,20)=3.85, p=.064$. The 
Age X Strategy interaction resulted from older adults making more gazes compared to young adults when computing, $t(20)=2.17, p=.035, d=0.87$, but not when retrieving, $t(20)=0.12, p=.909 .{ }^{4}$ The Length X Strategy trend indicated a greater increase in gazes by string length for the computation strategy compared to the retrieval strategy. This latter trend also supports the validity of the strategy reports as longer strings require more gazes for computation relative to smaller strings whereas they do not require more gazes to retrieve (where only the first and last letters need to be gazed regardless of string length).The three way interaction was not reliable, $F<1$. Thus, the validity of retrieval strategy reports was supported by fewer gazes overall and smaller addend effects when retrieving compared to computing.

Selective attention condition: Non-triplet gaze counts for the selective attention condition are shown in Figure 3 (right panel). The main effect of age was not reliable, $F(1,19)=1.33$, $p=.264$. As predicted, a main effect of strategy, $F(1,19)=50.11, p<.001, d=1.34$, resulted from more gazes outside the triplets for computation compared to selective attention. A main effect of length, $F(1,19)=57.85, p<.001, d=0.88$, resulted from more gazes on longer strings. However these main effects were qualified by a predicted Strategy $\mathrm{X}$ Length interaction, $F(1,19)=16.61, p<.001$. This resulted from a greater increase in gaze counts for longer strings when computation was used compared to when selective attention was used, $t(28)=3.31, p=.003, d=1.17$. The string length effect was reduced but still reliable when selective attention was used, $t(19)=1.79, p=.005, d=0.58$. The threeway interaction was not reliable, $F<1$.

When selective attention was reported the mean number of gazes to the non-triplet portion of the string was still reliably greater than zero $(M=1.52, S E=0.33), t(19)=3.81, p=.001$. Also important for validating strategy reports, the mean number of gazes for +2 and +4 strings when using selective attention were $1.00(S E=0.27)$ and $2.00(S E=0.56)$ respectively, which is almost certainly insufficient for processing all non-triplet letters. In contrast, the mean number of gazes for +2 and +4 strings when computation was used were $2.82(S E=0.35)$ and $6.15(S E=0.55)$, more than enough to process the entire non-triplet region. Thus, selective attention reports do appear to be generally valid for both young and older adults.

Response time benefits-Response time data can be found in Table 1. Median RTs were examined via 2 (age: young, old) X 2 (strategy: computation, retrieval/selective attention) repeated measures GLMs. ${ }^{5}$ Participants excluded from gaze analyses due to poor capture were retained for RT analyses.

For the retrieval condition, a main effect of age, $F(1,26)=10.82, M S E=40,891,943, p=$. $002, d=0.52$, resulted from older adults taking longer to respond. A main effect of strategy, $F(1,26)=104.62, M S E=387,439,530, p<.001, d=3.86$, resulted from longer RTs for computation compared to retrieval. The Age $\mathrm{X}$ Strategy interaction was not reliable, $F(1,26)$ $=1.43, M S E=5,281,782, p=.243$. For the selective attention condition, the main effect of age, $F(1,31)=2.54, M S E=20,817,822, p=.121$, was not reliable. A main effect of strategy, $F(1,31)=23.35, M S E=99,711,140, p<.001, d=0.91$, resulted from longer RTs

\footnotetext{
${ }^{4}$ Older adults' greater and more variable gaze counts when reporting computation in the retrieval condition appears to be driven in part by outliers who used computation infrequently. Because older adults made more gazes compared to young adults when computing but not when retrieving, older adults exhibited a greater reduction in gaze counts when shifting to retrieval. It is unclear why older adults made more gazes compared to young when computing in this condition; no age difference in computation gaze counts was found in Phase 1 or in the selective-attention condition, so this outcome will not be considered further.

5 Response times were also examined by length. The results generally mimic the pattern seen in gaze data with longer RTs for longer strings when computing but not when retrieving or using selective attention.
} 
for computation compared to selective attention. The Age X Strategy interaction just missed significance, $F(1,31)=3.79, M S E=16,208,208, p=.061$.

In summary, selective attention and retrieval were more efficient (faster) compared to computation, supporting the validity of the strategy reports. Furthermore, a between-subjects $t$-test showed that retrieval was considerably faster than selective attention, $t(76)=12.55, p$ $<.001, d=2.74$, which may in part explain older adults' general tendency to choose retrieval over selective attention and computation in the choice condition (the analogous test in the choice condition was ill-powered due to infrequent selective attention use). Faster RTs for selective attention and retrieval compared to computation also support the validity of the strategy reports.

\section{Metacognitive ratings}

Following Phase 3, participants rated each available strategy in terms of 1) their confidence in their ability to use it, 2) how difficult they found it, and 3) how much they felt it improved performance speed, with each rating made on a $0-100$ scale (100 being very confident/very difficult/much faster). In the interest of brevity, only effects reliable at the .05 level are reported.

For the retrieval and selective attention conditions, ratings were examined using a series of 2 (age: young, old) X 2 (condition: retrieval, selective attention) ANOVAs (see Figure 4 left panel). Age X Condition interactions for confidence, $F(1,76)=5.95, M S E=5,158, p=$. 017 , and difficulty ratings, $F(1,76)=4.70, M S E=2,850, p=.033$, resulted from young adults in the selective attention condition rating themselves less confident and rating selective attention more difficult compared to both young and older adults in the retrieval condition and compared to older adults in the selective attention condition; no such differences were reported by older adults ( $p s<.05$ for noted young adult contrasts; all other $p \mathrm{~s}>$.2). Both young and older adults rated retrieval as being faster than selective attention, $F(1,76)=18.00, M S E=12,476, p<.001, d=0.95$.

In the choice condition, confidence, difficulty, and perceived performance benefits were examined using a series of 2 (age: young, old) X 2 (strategy: retrieval, selective attention) ANOVAs (see Figure 4). The selective attention strategy was rated more difficult, $F(1,40)=$ 7.07, $M S E=5,563, p=.011, d=0.56$, and to have less speed benefit, $F(1,40)=224.64$, $M S E=108,822, p<.001, d=3.53$, and participants were marginally less confident in selective attention use, $F(1,40)=3.91, M S E=3,381, p=.055$. Thus, young adults rated selective attention less favorably compared to alternative strategies in both the choice and selective attention conditions, whereas older adults rated selective attention less favorably only in the choice condition when a more efficient strategy (retrieval) was available.

\section{Metacognitive correlates of strategy use}

The correlations between individual differences in strategy use and metacognitive strategy ratings are reported in Table 2. Participants were more likely to use a strategy they were confident in, and this was generally true for young and older adults for both retrieval and selective attention (other than an unreliable correlation between selective attention use and confidence for older adults, $p=.110$ ). Young and older adults were also more likely to use a strategy they felt was less difficult. However, only older adults were more likely to use a strategy if they felt it improved performance speed. ${ }^{6}$

\footnotetext{
${ }^{6}$ Although the overall correlation for young adults' strategy use and speed was reliable, this appears to be an artifact of retrieval having been used more and rated higher compared to selective attention with no correlation between use and speed within either strategy for young adults $(p s>.250)$.
} 
Because participants in the choice condition had two alternative strategies to choose from, it stands to reason that one's impression of the retrieval strategy relative to the selective attention would play a role in which of those strategies they chose to engage in. Thus, for the choice condition, we examined how within-subject differences in use of the retrieval and selective attention strategies correlated with within-subject differences in strategy ratings. Each participant's percent selective attention use was subtracted from their retrieval use. Thus, participants with positive scores used retrieval more than selective attention, and participants with negative scores used selective attention more than retrieval. Analogous differences scores were computed for each participant's strategy ratings of confidence, difficulty, and speed.

Table 2 presents the correlations between use differences and rating differences. Participants who were more confident in their ability to use retrieval versus selective attention used retrieval more than selective attention. Young adults who rated retrieval as less difficult compared to selective attention were more likely to use retrieval, although this was not the case for older adults $(p=.095)$. Perceived benefits of performance speed were positively correlated with strategy choice for older adults, but not young adults $(p=.134)$, consistent with the pattern of correlations in the retrieval and selective attention conditions. To clarify, it is important to note that no participants rated the selective attention strategy faster than the retrieval strategy. However, those older adults who judged the speed benefit of retrieval to be smaller relative to selective attention chose to use selective attention more often. Thus, older adults who perceived less of a discrepancy in the speed of executing the two strategies were less likely to opt for the faster retrieval strategy. However, an inspection of the raw data indicates that a few older adults did recognize the benefits of the retrieval strategy and still chose to compute, perhaps to avoid costs associated with switching or due to poor memory confidence.

\section{Discussion}

Our evidence clearly supports the retrieval reluctance hypothesis. Older adults manifested a relative reluctance to shift strategies that required intentional memory retrieval, but did not avoid shifting to a selective attention strategy. This evidence weighs against the general shift reluctance hypothesis, which predicts that older adults will be more likely to maintain initially established strategic processing habits in a task. Older adults in the selective attention condition adopted a non-retrieval-based selective attention strategy to a greater extent than did young adults.

The current experiment was the first to use item-level strategy reports to measure a selective attention strategy. It appears that participants could reliably monitor and validly report use of the selective attention strategy in this task, given the patterns of eye-tracking results and RTs for different reported strategies. Reported computation trials showed more non-triplet gazes for longer strings, retrieval trials showed fewer gazes overall, and selective attention trials showed smaller increases in gaze counts for longer strings. Although the string length effect was still reliable with selective attention (see also Haider \& Frensch, 1999) participants may reduce but not fully eliminate gazes to irrelevant portions of the string, which can be relatively automatic (Touron et al., 2010). Haider and Frensch (1999) also suggested that some participants may use the first letters to facilitate computation of the triplet, while still ignoring the majority of the irrelevant information. Response times on reported retrieval trials were substantially faster than computation RTs, and selective attention RTs were faster than computation RTs and slower than retrieval RTs. All of these outcomes would be expected given the processing requirements of the different strategies, thereby supporting the validity of the item-level strategy reports. 
Although the typical finding of older adult retrieval reluctance was found, this effect was reduced in magnitude compared to previous studies, probably in large part because of our prelearning manipulation designed to clarify strategic choice behavior. Prelearning is known to increase older adult retrieval use (Hines et al., 2012; Touron \& Hertzog, 2004a). Older adults' retrieval use may have also been greater in the current study because the AVT computation algorithm was procedurally simple but much slower and more effortful relative to the retrieval strategy. Touron and Hertzog's (2004b) noun-pair lookup study showed that increasing the display size and lowering the efficiency of visual scanning hastened older adults' shift to the retrieval strategy. In the current study using the AVT task, older adults' RT benefit for using retrieval was roughly $5 \mathrm{~s}$, whereas the RT benefit in previous work using the noun-pair task has typically been less than $2 \mathrm{~s}$ (e.g., Touron, 2006; Touron \& Hertzog, 2009).

We found relatively low rates of selective attention use among young adults. Although aggregate data suggest that Haider and Frensch's $(1996,1999)$ samples spontaneously adopted selective attention, strategy use was inferred from RT data rather than strategy reports, and stimuli were repeated roughly eight times, once every 25-105 trials, for a total of 200-800 trials, depending on the experiment. Thus, mixed retrieval strategy use may have contributed to their observed RT improvements (but see Haider \& Frensch, 1996,

Experiment 3). Younger adults in our study may have shifted less completely to the selective attention strategy due to their smaller amount of training time. Participants in the current study also received practice with the retrieval strategy during prelearning, and instructions but not explicit practice with the selective attention strategy, which may have biased them in favor of the retrieval strategy. Lastly, younger adults' eye-movements were less distinct in terms of gaze counts between the computation and selective attention strategies compared to older adults. Thus, some young adults may have struggled to differentiate these two strategies, whereas older adults did not appear to have this problem.

Our metacognitive ratings data suggest that young adults were unwilling to use a strategy that they felt was more difficult or in which they lacked confidence, regardless of whether or not they felt that the strategy would have been faster. Perhaps some young adults found attending to the characters preceding the non-triplet region either difficult to ignore or beneficial to performance. In contrast, older adults readily switched to the selective attention strategy, even more so when they believed the selective attention strategy to be faster and less difficult.

Nevertheless, older adults in the choice condition typically chose the retrieval strategy over selective attention. A possible explanation for this pattern is that the availability of multiple strategic options encouraged evaluation of relative costs and benefits of the two alternative strategies to the standard algorithm. In the choice condition, selecting the retrieval strategy over selective attention was correlated with whether older adults felt more confident in the retrieval strategy versus the selective attention strategy, and whether they found retrieval to be substantially faster compared to selective attention. No participant in the current study rated the selective attention strategy to be faster than the retrieval strategy. However, older adults varied in perceived retrieval efficiency, and manifested a reluctance to use it if they rated it as minimally faster than selective attention. This suggests that older adults are particularly avoidant of retrieval-based strategies unless sufficiently confident and/or motivated by perceived performance benefits (Touron \& Hertzog, 2009). These findings are consistent with previous evidence for a RT monitoring deficit contributing to older adults' reluctance to use retrieval-based strategies in the noun-pair lookup task (Hertzog et al., 2007). Alternatively, older adults' lack of confidence in the retrieval strategy may indicate that they did not believe themselves capable of using retrieval despite having achieved greater than $90 \%$ accuracy during prelearning. Consistent with this interpretation, Hines et 
al. (2012) demonstrated that age differences in retrieval use can be eliminated given more stringent prelearning criteria.

The current research rules out a general shift reluctance hypothesis in standard skill acquisition tasks where holistic strategy shift is possible; however, this may not generalize to tasks where multiple switches are required for optimal performance. Terry and Sliwinski (2012) demonstrated that older adults performing voluntary task switching are more likely to repeat the same task on successive trials, and interpret this repetition as an avoidance of costs they incur from maintaining multiple task sets in working memory. Likewise, Lemaire and Lecacheur (2010) have shown that young adults may be reluctant to shift strategies based on differential strategy affordances that vary from trial to trial, possibly due to the added switch costs. Our task did not require multiple strategy shifts to achieve optimal performance (holistic strategy shift was both possible and optimal given prelearning and strategy instruction). Older adults may be less willing to shift in tasks where the optimal approach involves shifting strategies from trial to trial depending on item characteristics (Lemaire \& LeClere, 2012). Consistent with this perspective, Touron (2006) found that while young adults shift to retrieval at an item level, older adults tend to shift to retrieval more holistically across an entire item set.

We acknowledge that the current study examines strategy shift in a task with limited strategic options - there is only one possible alternative strategy to memory retrieval other than full computation. It is unknown how older adults would shift strategic behaviors in even more complex tasks. It also may be the case that older adults are particularly willing to shift to selective attention or other "step-skipping" versions of previous strategies but not to entirely new strategies. A task where one can compute the solution, look-up the solution, or retrieve it from memory would help identify if older adults are willing to shift to new strategy that is not merely an alternative version of a familiar algorithm; for example, an everyday task with analogous strategy alternatives might allow mentally computing the amount to tip for a regularly ordered meal, using a calculator, or remembering how much you tipped on previous visits. Although there is currently no evidence that selective attention strategies should be particularly attractive to older adults, future research should consider shift-reluctance in other non-retrieval skill acquisition tasks. Given the current results, it seems probable that the particular set of affordances for available strategies should be more impactful on strategy choice than any global resistance by older adults to shift task approach. Future research should also consider manipulating the cost-benefit tradeoff of selective attention shift to retrieval shift to discover whether young adults might adopt the strategy more if it were more beneficial or if older adults might adopt it less if it were less beneficial.

In summary, the present study shows that older adults manifest a reluctance to shift to retrieval based strategies, but one that is not universal. They will shift to retrieval if it is perceived as being much more efficient and effective than competing alternatives. Thus, their retrieval reluctance can be framed as a biased strategic preference, one grounded in a greater perceived risk of retrieval failures and a reduced perception of retrieval benefits for RTs.

\section{Acknowledgments}

This research was supported by a National Institute on Aging grant, NIA R01 AG024485. The authors would like to extend special thanks to Amanda Brewer for her assistance with subject recruitment and data collection. 


\section{References}

Anderson J, Matessa M, Lebiere C. ACT-R: A theory of higher level cognition and its relation to visual attention. Human-Computer Interaction. 1997; 12:439-462.

Arrington CM, Logan GD. The cost of a voluntary task switch. Psychological Science. 2004; 15:610615. [PubMed: 15327632]

Arrington CM, Logan GD. Voluntary task switching: Chasing the elusive homunculus. Journal of Experimental Psychology: Learning, Memory, and Cognition. 2005; 31:683-702.

Bourne LE, Raymond WD, Healy AF. Strategy selection and use during classification skill acquisition. Journal of Experimental Psychology: Learning, Memory, \& Cognition. 2010; 36(2):500-514.

Cerella J, Onyper SV, Hoyer WJ. The associative memory basis of cognitive skill learning: Adult age differences. Psychology \& Aging. 2006; 21:483-498. [PubMed: 16953711]

Dunlosky J, Hertzog C. Measuring strategy production during associative learning: The relative utility of concurrent versus retrospective reports. Memory \& Cognition. 2001; 29(2):247-253. [PubMed: 11352207]

Frensch, PA.; Sternberg, RJ. Skill-related differences in game playing. In: Sternberg, RJ.; Frensch, PA., editors. Complex problem solving: Principles and Mechanisms. Hilldale, NJ: Erlbaum; 1991. p. 334-381.

Haider H, Frensch PA. The role of information reduction in skill acquisition. Cognitive Psychology. 1996; 30(3):304-337. [PubMed: 8660787]

Haider H, Frensch PA. Eye movement during skill acquisition: More evidence for the informationreduction hypothesis. Journal of Experimental Psychology: Learning, Memory, and Cognition. 1999; 25(1):172-190.

Haider H, Frensch PA, Joram D. Are strategy shifts caused by data-driven processes or by voluntary processes? Consciousness and Cognition. 2005; 14:495-519. [PubMed: 16091268]

Hertzog C, Price J, Dunlosky J. Age differences in the effects of experimenter instructed versus selfgenerated strategy use. Experimental Aging Research. 2012; 38:42-62. [PubMed: 22224949]

Hertzog C, Touron DR. Age differences in memory retrieval shift: Governed by feeling-of-knowing? Psychology and Aging. 2011; 26(3):647-660. [PubMed: 21401263]

Hertzog C, Touron DR, Hines J. Does a time monitoring deficit influence older adults' delayed retrieval shift during skill acquisition? Psychology and Aging. 2007; 22:607-624. [PubMed: 17874958]

Hines J, Hertzog C, Touron DR. A prelearning manipulation falsifies a pure associational deficit account of retrieval shift during skill acquisition. Aging, Neuropsychology, and Cognition. 2012; 19:449-478.

Hoyer WJ, Cerella J, Onyper SV. Item learning in cognitive skill training: Effects of item difficulty. Memory \& Cognition. 2003; 31:1260-1270. [PubMed: 15058687]

Kausler, DH. Learning and memory in normal aging. Sam Diego, CA: Academic Press; 1994. 1994-97905-000

Kramer AF, Hahn S, Gopher D. Task coordination and aging: Explorations of executive control processes in the task switching paradigm. Acta Psychologica. 1999; 101:339-378. [PubMed: 10344190]

Lamson N, Rogers WA. Assessing age-related patterns in strategy selection on a mathematical problem-solving task. The Journals of Gerontology: Series B: Psychological Sciences and Social Sciences. 2008; 63B(3):146-155.

Lemaire P. Cognitive strategy variations during aging. Psychological Science. 2010; 19(6):363-369.

Lemaire, P.; LeClere, M. Aging and strategy repetition during cognitive tasks; Poster presented at the 2012 Cognitive Aging Conference; Atlanta, GA. Apr. 2012

Lemaire P, Lecacheur M. Strategy switch costs in arithmetic problem solving. Priming processes may underlie strategy switch costs. Memory and Cognition. 2010; 38(3):322-332. [PubMed: 20234022]

Littell, RC.; Milliken, GA.; Stroup, WW.; Wolfinger, RD. SAS system for mixed models. 4th ed.. Cary, NC: SAS Institute, Inc; 2000. 
Mayr U. Age differences in the selection of mental sets: The role of inhibition, stimulus ambiguity, and response-set overlap. Psychology and Aging. 2001; 16:96-109. [PubMed: 11302371]

Ratcliff R, Thapar A, McKoon G. Effects of aging and IQ on associative memory. Journal of Experimental Psychology: General. 2011; 140:464-487. [PubMed: 21707207]

Rawson KA, Touron DR. Age differences and similarities in the shift from computation to retrieval during reading comprehension. Psychology and Aging. 2009; 24(2):423-437. [PubMed: 19485659]

Rogers WA, Gilbert DK. Do performance strategies mediate age-related differences in associative learning? Psychology and Aging. 1997; 12(4):620-633. [PubMed: 9416631]

Rogers WA, Hertzog C, Fisk AD. An individual differences analysis of ability and strategy influences: Age-related differences in associative learning. Journal of Experimental Psychology: Learning, Memory, \& Cognition. 2000; 26:359-394.

Siegler RS. Strategy choice procedures and the development of multiplication skills. Journal of Experimental Psychology: General. 1988; 117:258-275. [PubMed: 2971762]

Spieler DH, Mayr U, LaGrone S. Outsourcing cognitive control to the environment: adult age differences in the use of task cues. Psychonomic Bulletin \& Review. 2006; 13:787-793. [PubMed: 17328374]

Strayer DL, Kramer AF. Aging and skill acquisition: learning-performance distinctions. Psychology and Aging. 1994; 9(4):589-605. [PubMed: 7893430]

Touron DR. Are item-level strategy shifts abrupt and collective? Age differences in cognitive skill acquisition. Psychonomic Bulletin \& Review. 2006; 13:781-786. [PubMed: 17328373]

Touron DR, Hertzog C. Age differences in strategic behavior during a computation-based skill acquisition task. Psychology and Aging. 2009; 24(3):574-585. [PubMed: 19739913]

Touron DR, Hertzog C. Distinguishing Age Difference in Knowledge, Strategy use, and confidence during strategic skill acquisition. Psychology and Aging. 2004a; 19(3):452-466. [PubMed: 15382996]

Touron DR, Hertzog C. Strategy shift affordance and strategy choice in young and old adults. Memory \& Cognition. 2004b; 32:298-310. [PubMed: 15190721]

Touron DR, Hertzog C, Frank D. Eye movements and strategy shift in skill acquisition: Adult age differences. Journal of Gerontology: Psychological Sciences. 2010; 66B(2):151-159.

Touron DR, Hoyer WJ, Cerella J. Cognitive skill acquisition and transfer in younger and older adults. Psychology and Aging. 2001; 16:555-563. [PubMed: 11766911]

Touron DR, Hoyer WJ, Cerella J. Cognitive skill acquisition and transfer in younger and older adults. Psychology and Aging. 2001; 16:555-63. [PubMed: 11766911]

Touron DR, Swaim E, Hertzog C. Moderation of older adults' retrieval reluctance through task instructions and monetary incentives. Journals of Gerontology, Series B: Psychological Sciences and Social Sciences. 2007; 62:149-155.

Wechsler, D. Wechsler Adult Intelligence Scale—Revised. New York: Psychological Corporation; 1981.

Zachary, RA. Shipley Institute of Living Scale: Revised manual. Los Angeles: Western Psychological Services; 1986.

\section{APPENDIX}

\begin{tabular}{|c|c|}
\hline Phase 2 Correct Strings & $\underline{\text { How they appeared at test }}$ \\
\hline B C D E F [4] K & $\mathrm{B}_{----}[?] \mathrm{K}$ \\
\hline D E F G H [5] N & $\mathrm{D}_{----}[?] \mathrm{N}$ \\
\hline F G H [4] M & $\mathrm{F}_{--}[?] \mathrm{M}$ \\
\hline H I J [5] P & $\mathrm{H}_{--}[?] \mathrm{P}$ \\
\hline G [4] L & $\mathrm{G}[?] \mathrm{L}$ \\
\hline
\end{tabular}

Psychol Aging. Author manuscript; available in PMC 2014 September 01. 


\begin{tabular}{cc} 
I [5] O & I [?] O \\
Sample Phase 2 Incorrect Strings & How they appeared at test \\
\cline { 2 - 2 } B D E F G [4] L & B $_{---}[$? ] L \\
D E F G H [5] M & $\mathrm{D}_{----}[$? ] M \\
F H I [4] N & F $_{--}[?] \mathrm{N}$ \\
H I J [5] O & $\mathrm{H}_{--}[$? ] O \\
G [4] K & G [?] K \\
I [5] P & I [?] P \\
\hline
\end{tabular}

Psychol Aging. Author manuscript; available in PMC 2014 September 01. 


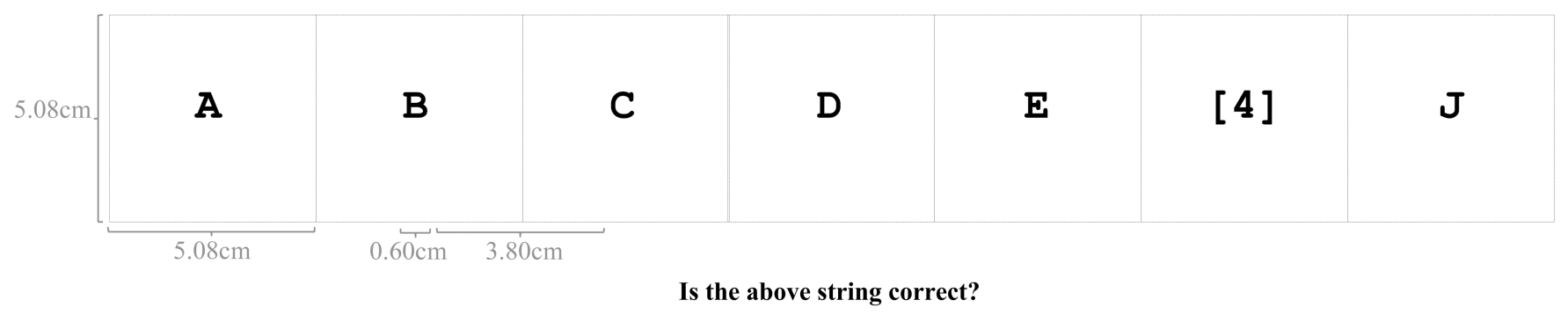

\section{Yes $=\mathbf{Y} \quad$ No $=\mathbf{N}$}

Figure 1.

Screen shot of AVT task with superimposed distances and areas of interest (AOIs). 


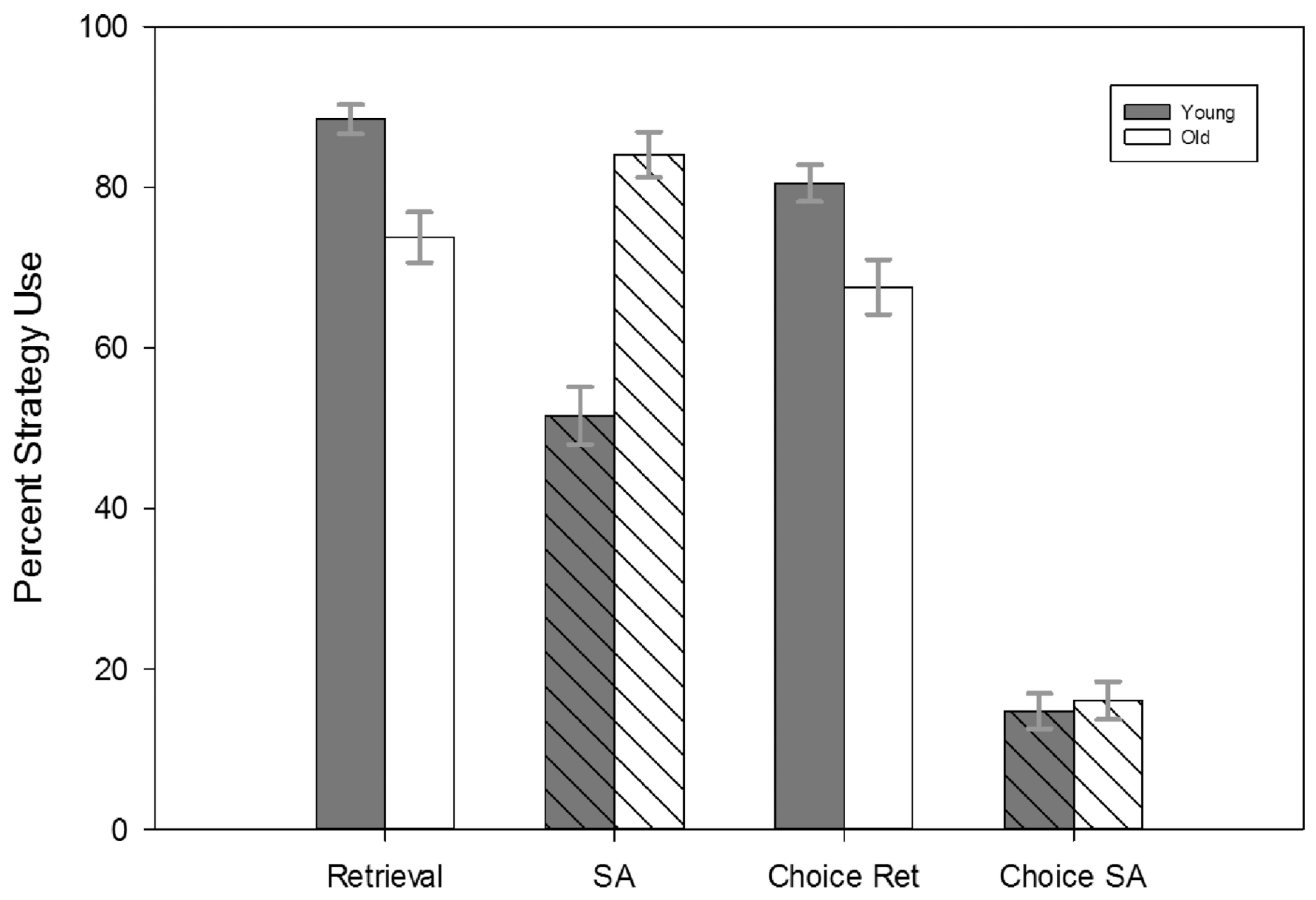

Figure 2.

Phase 3 percentage of retrieval and selective attention use (strategy use) and standard error bars by age and condition. Choice Ret = retrieval strategy use in the choice condition; Choice $\mathrm{SA}=$ selective attention use in the choice condition. 
Retrieval Condition

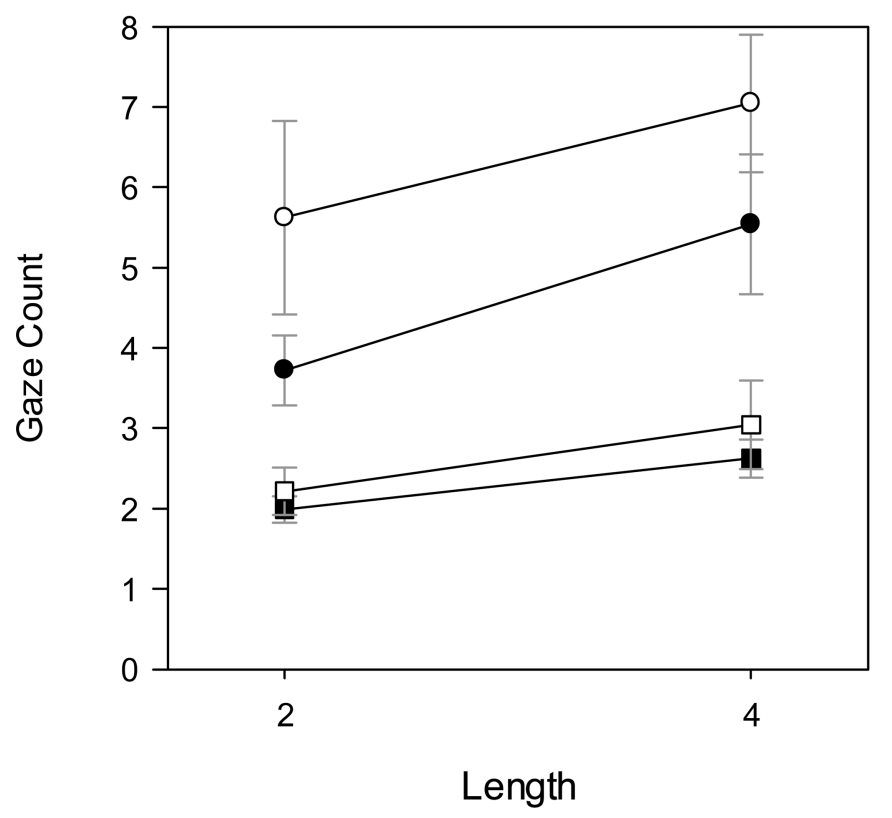

Selective Attention Condition

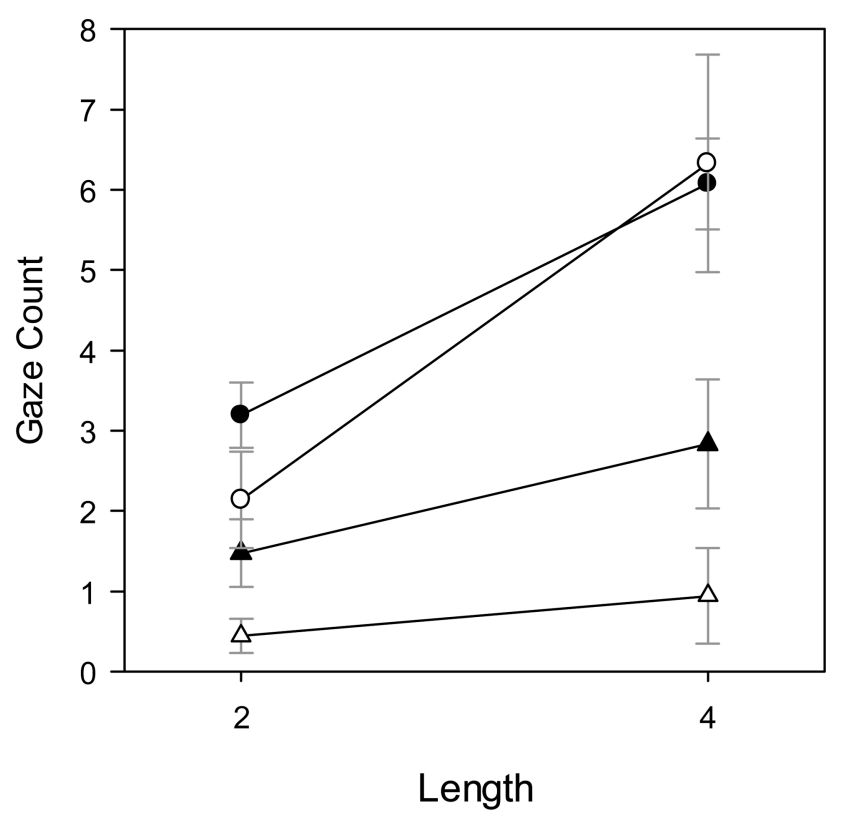

\section{Figure 3.}

Phase 3 retrieval condition gaze counts to the non-triplet by age and length. Left Panel:

Retrieval condition. Right Panel: Selective attention condition. Length refers to the number of letters in addition to the triplet. Ret $=$ retrieval; Comp $=$ computation; $\mathrm{SA}=$ selective attention. 

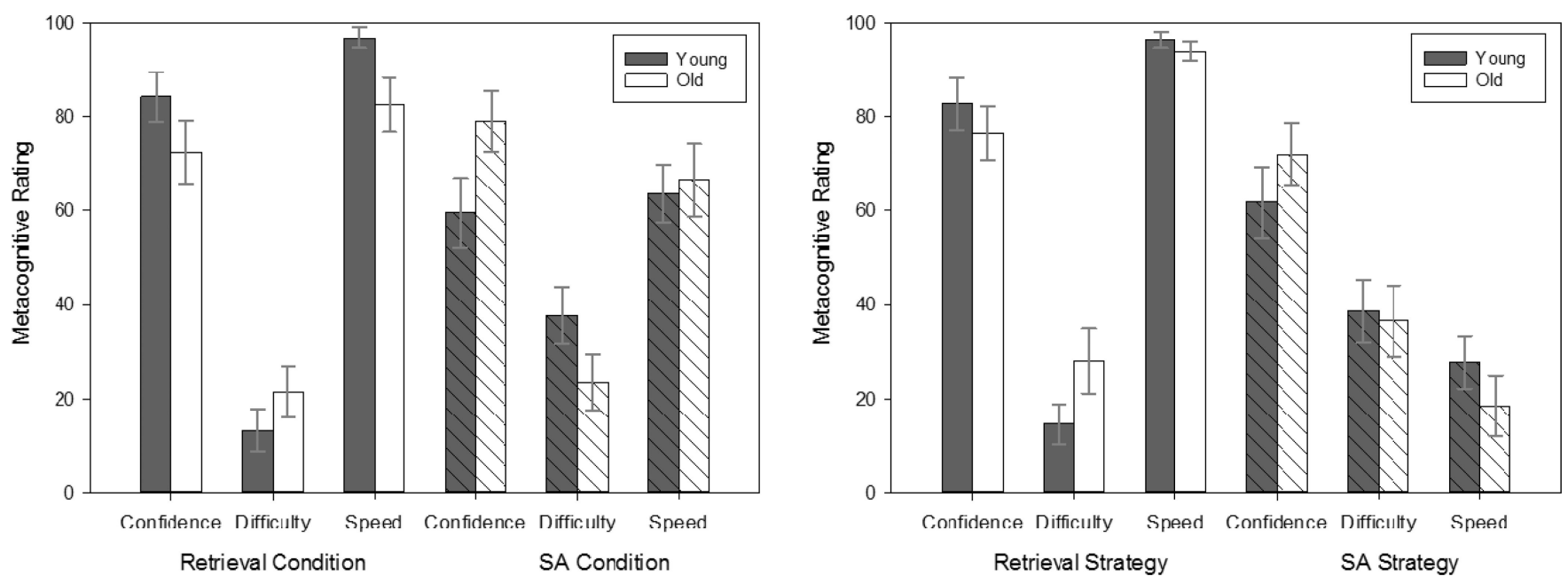

Figure 4.

Metacognitive ratings. Left Panel: Retrieval and selective attention conditions. Right Panel: Choice condition. 
